\title{
Avant-propos
}

\section{Entre reconstruction et mutations, les industries de la chimie entre les deux guerres}

L'après Première guerre mondiale a favorisé le déclenchement de la professionnalisation de la chimie en France, tandis que l'industrie chimique se recomposait dans un cadre économique profondément modifié par le conflit.

L'ouvrage présente en premier lieu des organisations nationales et internationales de la chimie. En particulier, des réseaux très actifs de chimie industrielle ont contribué à renforcer les échanges entre spécialistes et à améliorer l'image du secteur. Avec leurs associations représentatives, les Français ont cherché à être mieux reconnus et à défendre la place de leur métier au sein des secteurs de l'industrie.

Parallèlement, les modifications profondes des activités industrielles survenues avec l'effort de guerre ont généré la production massive de différents produits chimiques et le développement de nouvelles entreprises. Est apparue aussi la nécessité de poursuivre et développer les recherches initiées durant la guerre.

Dans ce contexte, les évolutions observées dans différentes zones économiques du pays et dans plusieurs domaines de production sont abordées. Ainsi les restructurations des industries chimiques et des formations professionnelles dans le Sud-Est de la France sont examinées dans le cadre de la région lyonnaise. Pour élargir le 
panorama vers d'autres régions, vient ensuite la présentation d'initiatives industrielles prises dans l'Ouest (Nantes), le Sud-Ouest (Bordeaux), et le Midi (Toulouse). Enfin, l'étude d'une innovation, qui s'est expatriée aux États-Unis pour se développer avec succès, au moment de la recherche en France sur le « carburant national », est un exemple significatif des hésitations de la chimie dans l'entre-deuxguerres.

Développement ou stagnation des activités, tel est l'un des constats faits lors de ce parcours dans une période historique encore peu explorée.

G. Emptoz, D. Fauque, J. Breysse (éds.) 